\title{
Note: A helical velocity selector for continuous molecular beams
}

\author{
Carola Szewc, James D. Collier, and Hendrik Ulbricht ${ }^{a}$ \\ School of Physics and Astronomy, University of Southampton, Highfield, SO17 1BJ, United Kingdom
}

(Received 22 July 2010; accepted 17 September 2010; published online 19 October 2010)

\begin{abstract}
We report on a modern realization of the classic helical velocity selector for gas phase particle beams. The device operates stably under high vacuum conditions at rotational frequencies limited only by commercial dc motor capabilities. Tuning the rotational frequency allows selective scanning over a broad velocity band. The width of the selected velocity distributions at full-width-half-maximum is as narrow as a few percent of the selected mean velocity and independent of the rotational speed of the selector. The selector generates low vibrational noise amplitudes comparable to mechanically damped state-of-the-art turbo-molecular pumps and is therefore compatible with vibration sensitive experiments like molecule interferometry. (C) 2010 American Institute of Physics. [doi:10.1063/1.3499254]
\end{abstract}

Manipulation and control of electrically neutral particles, such as atoms and molecules in the gas phase is an active field of research. ${ }^{1}$ The general aim is to increase the ability to control the motion of particles as they propagate in gas phase beams by means of deceleration, ${ }^{2-5}$ trapping, ${ }^{6}$ and cooling. ${ }^{7,8}$ Today, cold atoms and small molecules are the best controlled physical systems and therefore are the ideal playground to study physics ${ }^{9}$ and chemistry ${ }^{10,11}$ at the most fundamental level. ${ }^{12}$

For more complex systems-starting with molecules of more than about ten atoms - this level of control has not yet been achieved. Velocity selection is one of the first steps to increase control over the motion of large molecules and clusters. For instance, molecule interferometry is challenging today's gas phase manipulation technologies and will gain from improved beam monochromaticity: by reaching maximal interference visibility, ${ }^{13}$ by higher precision in interferometric deflection for molecule metrology, ${ }^{14}$ as well as by interferometric particle sorting. ${ }^{15}$ Cooling and trapping of molecules will benefit from improved molecule velocity selection. ${ }^{12}$ Plainly, our device can be used to select slow particles from a wide thermal velocity distribution ${ }^{16}$ as proposed in one of the very early approaches to produce cold atoms. ${ }^{17}$ As a further example, velocity selection can be used to separate buffer gas cooled molecules thermalized with their lighter coolant atoms propagating at much higher velocities. Furthermore, atom, molecule and cluster lithography techniques ${ }^{18-20}$ will benefit from velocity pre-selected particles.

We here have picked up the old idea to mechanically separate particles of different velocities, for instance generated by a thermal beam source with a very broad velocity distribution and base our design on earlier helical velocity selectors for neutrons, ${ }^{21,22}$ atoms, ${ }^{23}$ and the related slotted disk selectors for molecules. ${ }^{24,25}$ The main idea is to make use of the difference in propagation time particles need for their passage through a spatial confinement. Such a confinement can be realized by a sequence slits arranged by rotating

\footnotetext{
${ }^{a)}$ Electronic mail: h.ulbricht@soton.ac.uk.
}

slotted disks at well defined positions and with well defined phase relations. It turns out that at least six such slotted disks are needed to realize velocity side-band free selection. The continuation to many slotted disks directly attached to each other is the helical velocity selector presented here. It consists of a metal cylinder with helical grooves on the outer surface. By rotating the cylinder, the path through the grooves is only open for molecules at a particular velocity. Here, we show a long-term stable operating helical velocity selector under high-vacuum conditions.

The working principle of the selector is best illustrated by its effect on a thermal velocity distribution, shown in Fig. 2. The device itself is shown in Fig. 1 and the theoretical description given summarizes earlier remarks by Dash et $a .^{21}$ The angle $\theta$ between the grooves and the cylinder axis is, for small angles, given by $\theta=r \phi / L$. When the cylinder rotates with angular velocity $\omega=2 \pi f=d \phi / d t$, the mean velocity of the selected molecules $v_{0}$-assuming a perfectly collimated molecular beam-is given by

$$
v_{0}=\frac{d L}{d t}=\frac{d L}{d \phi} \frac{d \phi}{d t}=\frac{L}{\phi} \omega
$$

where $L$ is the selector length and $L / \phi$ is the pitch. The velocity spread around $v_{0}$ is evaluated by calculating the minimal-maximal variations of the molecular beam path through a single groove at the given pitch angle $\phi_{\max , \min }$

$$
v_{\min , \max }=\frac{\omega L}{\phi_{\max , \min }}=\frac{\omega L}{\phi \pm \psi}=v_{0}\left(1 \pm \frac{\psi}{\phi}\right) .
$$

We therefore get a spread of the selected velocity distribution around $v_{0}$ at full-width-half-maximum (FWHM) of

$$
\frac{\Delta v}{v_{0}}(\mathrm{FWHM})=\frac{\psi}{\phi} \cdot \frac{1}{1-(\psi / \phi)^{2}},
$$

where the second term was neglected. We calculate the ideal resolution of $\Delta v / v_{0}(\mathrm{FWHM})=0.04$ for the selector parameter $\psi=9 \mathrm{mrad}$ and $\phi=0.22 \mathrm{rad}$ (see for a summary of all parameter of our realized selector Table I). Molecular beams are not perfectly collimated and the resolution changes to 


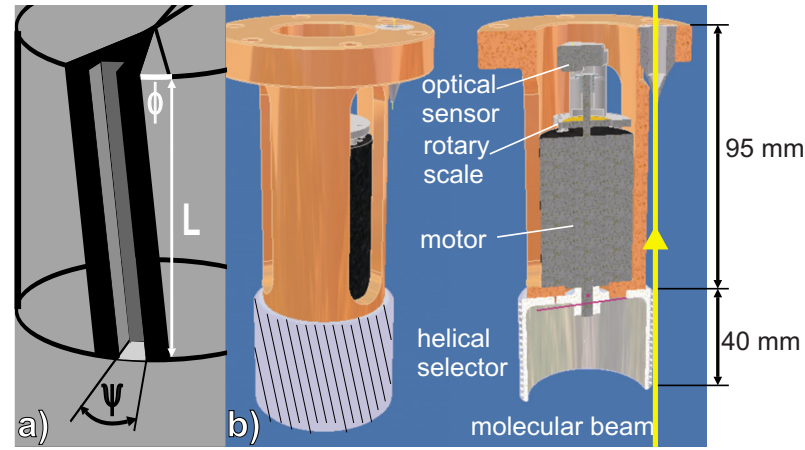

FIG. 1. (Color online) The measured width of the selected velocity distribution $\Delta v / v$ at full width half maximum from the time of flight measurements for different velocities. The dotted line is the theoretically expected value from Eq. (4). The inset shows the constant conversion of 1.13 between selector frequency and selected velocity. The TOF chopper was running at $80 \mathrm{~Hz}$.

$$
\frac{\Delta v}{v_{0}}(\mathrm{FWHM})=\frac{\psi+\alpha L / 2 r}{\phi},
$$

taking the divergence angle $\alpha$ into account. The theoretical resolution for a divergent beam of $\alpha=1 \mathrm{mrad}$ is therefore $\Delta v / v_{0}(\mathrm{FWHM})=0.044$.

The helical velocity selector as we use it in our molecular beam experiment is shown in Fig. 1. A rotating aluminum (alloy \#6082) cylinder has 430 grooves, $1 \mathrm{~mm}$ deep, on its outer surface, which was machined with a saw cutter attached to a four axis CNC mill. The width of the groove is $0.2 \mathrm{~mm}$ and the thickness of the bars is $0.15 \mathrm{~mm}$. The rotor was thermally shrink fitted directly to the motor axis to simplify the design and then balanced together with the brushless dc motor (Maxon, EC32, vacuum greased version) to reduce mechanical noise. Both, balancing and connecting the motor to a copper heat sink enabled operation over hours at temperatures around $45^{\circ} \mathrm{C}$. This temperature stability helped to maintain high vacuum conditions of 5 $\times 10^{-8}$ mbar during operation. The speed was detected by an ultrahigh vacuum compatible optical encoder module (GSI

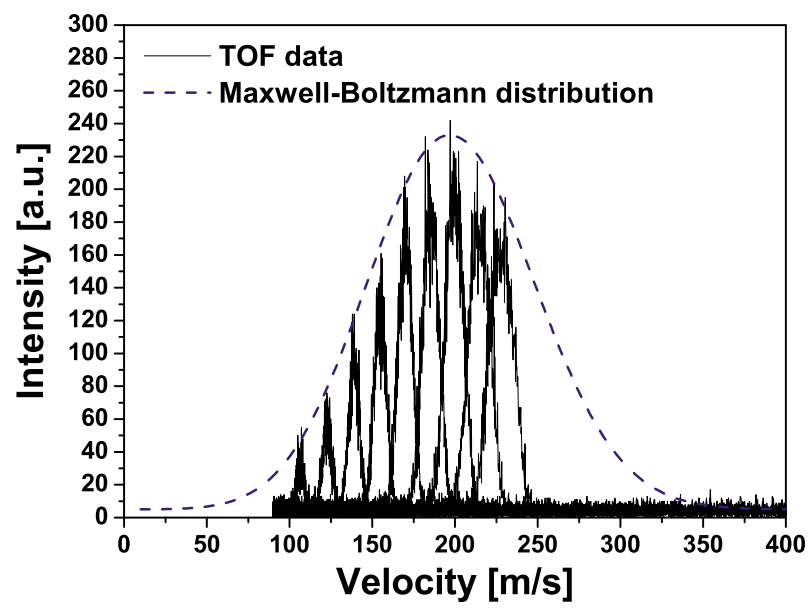

FIG. 2. (Color online) Schematic illustration of the selector. (a) gives details of a single groove on the outside of the cylinder. (b) shows the overall assembly: The rotor is directly mounted on the motor axis, which is thermally connected to the vacuum chamber by a copper rig and speed controlled by an optical encoder module. See Table I for dimensions of the selector.
TABLE I. The geometrical dimensions of the rotor of the velocity selector. The dimensions can be changed within the limits of mechanical precision engineering.

\begin{tabular}{lcc}
\hline \hline Radius of rotor & $r$ & $24 \mathrm{~mm}$ \\
Length of rotor & $L$ & $40 \mathrm{~mm}$ \\
Slit width & $d$ & $0.22 \mathrm{~mm}$ \\
Angular aperture of slits & $\psi=d / r$ & $9 \mathrm{mrad}$ \\
Groove angle & $\theta$ & $7.7^{\circ}$ \\
Pitch angle & $\phi=\theta L / r$ & $0.223 \mathrm{rad}$ \\
Divergence angle of molecular beam & $\alpha$ & $1 \mathrm{mrad}$ \\
\hline \hline
\end{tabular}

MicroE Systems, Mercury 1500V) and controlled by a servo amplifier module (Maxon, DES 50/5). Two straight grooves on the surface are used to align the selector to the molecular beam path. The rotation frequency was tuned up to $250 \mathrm{~Hz}$ and is limited by motor maximum speed and balancing of the motor-rotor assembly.

The theoretical conversion between the rotor frequency $f$ and the selected mean velocity of the molecules is $v_{0}$ $=1.13 f$ according to Eq. (1) for the given rotor dimensions (see Table I). The number of molecules passing the selector is $40 \%$ smaller than the initial beam intensity at the selected velocity and depends only on the open fraction of the selector, which is the ratio between groove width to the period of the grooves.

We experimentally characterize the velocity selector by using a vertically oriented time-of-flight (TOF) molecular beam setup. The molecular beam generated by a thermal source passes a chopper (Scitec, model 360C) before the helical selector and is then detected by a quadrupole mass spectrometer (Extrel, QMS9000) with an electron impact ionizer. The chopper gives the start signal for the TOF while the detector signal is the stop pulse analyzed by a digital measurements card (FastComtec, MCA-3 P7882). Selected molecule velocity distributions are extracted by deconvolution of the chopper function from the measured TOF signal. A series of velocity distributions analyzed from the measured TOF spectra is shown in Fig. 2. The distance be-

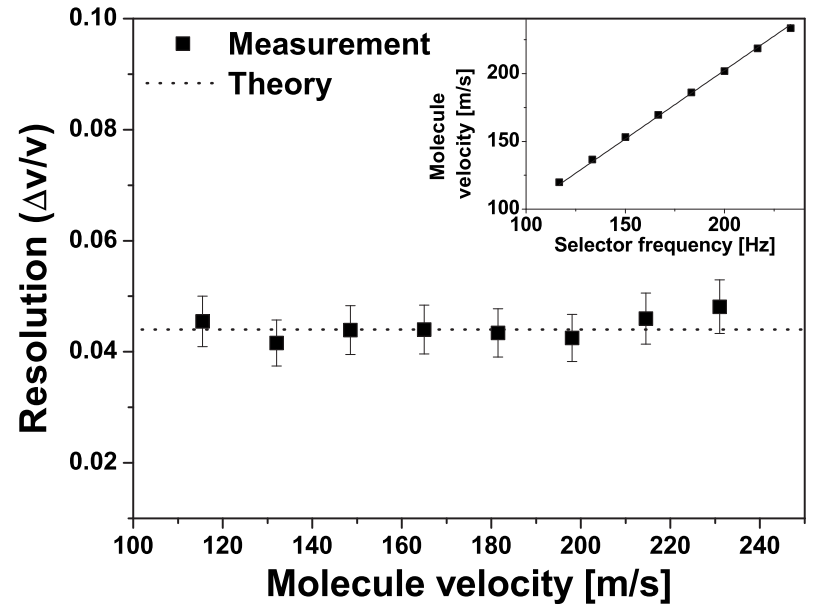

FIG. 3. Measured velocity distributions illustrating the function of the $\mathrm{v}$-selector for tetra-phenylporphyrin [TPP, mass is 614 atomic mass units $(\mathrm{amu})]$ molecules. The dashed line represents the Maxwell-Boltzmann distribution as generated by the thermal beam source at $570 \mathrm{~K}$. Time-of-flight measurements were taken and analyzed for different selector speeds. The TOF-chopper runs at $80 \mathrm{~Hz}$. 
tween chopper and channeltron detector was $1.38 \mathrm{~m}$. Additional time offset related to detection delays after ionization are small compared to the total flight times of $1 \mathrm{~ms}$ to $15 \mathrm{~ms}$ and are therefore not considered.

The envelope distribution of the thermal source can be described (dashed line in Fig. 2) by the floating MaxwellBoltzmann (fMB) distribution ${ }^{26}$

$$
f(v)=v^{2} \cdot \exp \left[-\frac{m\left(v-v_{m}\right)^{2}}{2 k_{b} T}\right]
$$

with the measured mean velocity $v_{m}$ (measure $)=167 \mathrm{~m} / \mathrm{s}$, which is faster than the expected mean velocity $v_{m}$ (expect) $=\sqrt{2 k_{b} T / m}=124 \mathrm{~m} / \mathrm{s}$ at the oven temperature of $570 \mathrm{~K}$. The spread of this fMB distribution corresponds to a molecule temperature of $T=217 \mathrm{~K}$. This points to the fact that our source is not operating fully within the effusive beam regime, in agreement with earlier observations. ${ }^{16}$ An acceleration due to supersonic expansion of the molecules at the oven nozzle with a diameter of $1 \mathrm{~mm}$ is in agreement with the observed higher mean velocity.

The selector rotation speed to molecule velocity conversion factor was evaluated to be 1.13 in perfect agreement with the theory and is shown in the inset of Fig. 3. The resolution of the velocity selector as extracted from the fullwidth-half-maximum of the de-convoluted TOF spectra and is in perfect agreement with the expected $4.4 \%$ for all measured velocities as shown in Fig. 3.

We investigate the mechanical noise generated by the selector in operation by attaching an accelerometer (Brühl\&Kjaer, type 4375 amplified by charge amplifier type $2635)$ to our vacuum chamber $(300 \mathrm{~kg}$ ) close to the point where the selector is attached. The vibration amplitudes are measured to be small compared to amplitudes generated by vibration damped turbo-molecular pumps running at around $1 \mathrm{kHz}$. We find amplitudes on the order of $1 \mathrm{~nm}$ to $10 \mathrm{~nm}$. We observe larger amplitudes at some specific rotational frequencies that might be in resonance with the setup eigenfrequencies and avoid running the selector at those during our measurements.

We have shown that a simple but careful design, based on commercially available parts, leads to a versatile helical velocity selector for molecular beams. Its rotational speed range, vacuum compatibility, and favorable vibrational characteristics make it a promising monochromator for challenging experiments as molecule interferometry, as well as many other applications using atomic, molecular or cluster beams. The particle velocity to be selected can be tuned by changing the rotational speed of the selector during operation and by adapting the geometry of the rotor-and here the slope of the grooves-during the design of the device. The width of the selected velocity distribution can be maintained by the groove width and the length of the rotor. Distributions of widths below one percent are possible, while challenging mechanical precision engineering. The reduction of the number of passing particles from the initial beam can be tuned by variation of the ratio between groove width to bar thickness. Balancing of the assembly, including motor and rotor, is essential to achieve small mechanical noise amplitudes making the device adaptable to vibration sensitive experiments. Future versions of the selector may utilize extreme condition dc motors to enable long lasting lifetime of the selector, which is limited by the evaporation time of the grease of the motor bearings. The compact design is more convenient to align and to balance compared to the slotted disk version and avoids velocity side bands.

In summary, in comparison to the earlier velocity selector designs we have improved its stable long term operation under high-vacuum conditions, we have significantly reduced vibration noise and realized a very compact design enabled by advanced mechanical precision engineering. This makes our device compatible to many modern molecular beam experiments.

We acknowledge excellent technical support by P. Connell and G. Savage. We thank S. Truppe, T. Juffmann, P. Geyer, A. G. Major, and M. Arndt for discussion.

${ }^{1}$ Interactions in Ultracold Gases, edited by M. Weidemuller and C. Zimmermann (Wiley-VCH, Weinheim, 2003).

${ }^{2}$ S. E. Maxwell, N. Brahms, R. deCarvalho, D. R. Glenn, J. S. Helton, S. V. Nguyen, D. Patterson, J. Petricka, D. DeMille, and J. M. Doyle, Phys. Rev. Lett. 95, 173201 (2005).

${ }^{3}$ H. L. Bethlem, G. Berden, and G. Meijer, Phys. Rev. Lett. 83, 1558 (1999).

${ }^{4}$ R. Fulton, A. Bishop, M. N. Shneider, and P. F. Barker, Nat. Phys. 2, 465 (2006).

${ }^{5}$ S. A. Rangwala, T. Junglen, T. Rieger, P. W. H. Pinkse, and G. Rempe, Phys. Rev. A 67, 043406 (2003).

${ }^{6}$ S. Y. T. van de Meerakker, H. L. Bethlem, and G. Meijer, Nat. Phys. 4, 595 (2008).

${ }^{7}$ S. Jochim, M. Bartenstein, A. Altmeyer, G. Hendl, S. Riedl, C. Chin, J. Hecker Denschlag, and R. Grimm, Science 302, 2101 (2003).

${ }^{8}$ S. Chu, L. Hollberg, J. E. Bjorkholm, A. Cable, and A. Ashkin, Phys. Rev. Lett. 55, 48 (1985).

${ }^{9}$ U. Schneider, L. Hackermuller, S. Will, Th. Best, I. Bloch, T. A. Costi, R. W. Helmes, D. Rasch, and A. Rosch, Science 322, 1520 (2008).

${ }^{10}$ S. Knoop, F. Ferlaino, M. Berninger, M. Mark, H.-C. Nägerl, R. Grimm, J. P. D'Incao, and B. D. Esry, Phys. Rev. Lett. 104, 053201 (2010).

${ }^{11}$ S. Ospelkaus, K.-K. Ni, D. Wang, M. H. G. de Miranda, B. Neyenhuis, G. Quemener, P. S. Julienne, J. L. Bohn, D. S. Jin, and J. Ye, Science 327, 853 (2010).

${ }^{12}$ Cold and Ultracold Molecules, Faraday Discussions Vol. 142, edited by E. Hinds, R. Grimm, G. Meijer, T. Softly, and W. Stwalley (RSC Publishing, Cambridge, 2009).

${ }^{13}$ S. Gerlich, L. Hackermüller, K. Hornberger, A. Stibor, H. Ulbricht, M. Gring, F. Goldfarb, T. Savas, M. Müri, M. Mayor, and M. Arndt, Nat. Phys. 3, 711 (2007).

${ }^{14}$ S. Gerlich, M. Gring, H. Ulbricht, K. Hornberger, J. Tüxen, M. Mayor, and M. Arndt, Angew. Chem., Int. Ed. 47, 6195 (2008).

${ }^{15}$ H. Ulbricht, M. Berninger, S. Deachapunya, A. Stefanov, and M. Arndt, Nanotechnology 19, 045502 (2008).

${ }^{16}$ S. Deachapunya, P. J. Fagan, A. G. Major, E. Reiger, H. Ritsch, A. Stefanov, H. Ulbricht, and M. Arndt, Eur. Phys. J. D 46, 307 (2008).

${ }^{17}$ N. Ramsey, Rev. Mod. Phys. 62, 541 (1990).

${ }^{18}$ T. Juffmann, S. Truppe, P. Geyer, A. G. Major, S. Deachapunya, H. Ulbricht, and M. Arndt, Phys. Rev. Lett. 103, 263601 (2009).

${ }^{19}$ R. J. Clark, T. R. Mazur, A. Libson, and M. G. Raizen, e-print arXiv: 1004.5581

${ }^{20}$ D. J. Asunskis, I. L. Bolotin, J. E. Haley, A. Urbas, and L. Hanley, J. Phys. Chem. C 113, 19824 (2009).

${ }^{21}$ J. Dash and H. Sommers, Rev. Sci. Instrum. 24, 91 (1953).

${ }^{22}$ D. Bally, E. Tarina, and P. Pirlogea, Rev. Sci. Instrum. 32, 297 (1961).

${ }^{23}$ R. Miller and P. Kusch, Phys. Rev. 99, 1314 (1955).

${ }^{24}$ H. Hostettler and R. Bernstein, Rev. Sci. Instrum. 31, 872 (1960).

${ }^{25}$ R. van Steyn and N. Vester, J. Phys. E 5, 691 (1972).

${ }^{26}$ Atomic and Molecular Beam Methods, edited by G. Scoles, D. Bassi, U. Buck, and D. Lain'e (University Press, Oxford, 1988). 